\title{
The Impact of Social Support on Employees' Subjective Well Being: The Mediating Role of Optimism
}

\author{
Hui-wen Bai ${ }^{1, a^{*}}$ \\ ${ }^{1}$ School of Business, Macau university of science and technology, Macau, China \\ a*baihuiwen619@163.com
}

\begin{abstract}
In this paper makes an empirical study on the relationship between social support and employee well-being, as well as the mediating role of optimism in psychological capital between social support and subjective well-being. Taking 311 employees from different industries in Shenzhen Special Economic Zone as the empirical research object, the research hypotheses are as follows: (1) Social support has a positive correlation with subjective well-being; (2) social support has a significant positive correlation with optimism; (3) optimism has a significant positive correlation with subjective well-being; (4) optimism plays a mediating role in the relationship between social support and subjective well-being, that is, social support can affect employees' subjective well-being through optimism. The results are of great significance to the theory and practice of social support, optimism and subjective well-being.
\end{abstract}

Keywords: Social support, Optimism, Subjective well-being

\section{社会支持对员工主观幸福感的影响一乐观的中介作用}

\author{
白惠文 ${ }^{1, a}$
}

\footnotetext{
澳门科技大学商学院，澳门，中国

a* baihuiwen_619@163.com
}

\section{摘要}

本文对社会支持与员工幸福感之间的关系，以及心理资本中的乐观在社会支持与主观幸福感的中介关系进行 了实证研究。研究以深圳特区不同行业的 311 名员工为实证研究对象, 通过问卷调查数据进行研究假设发现: （1）社会支持对主观幸福感的影响具有正相关;（2）社会支持对乐观具有显著正相关;（3）乐观对主观幸福 感具有显著正相关;（4）乐观在社会支持与主观幸福感关系中具有中介作用，即社会支持通过乐观能够影响员 工的主观幸福感。该研究结果对社会支持、乐观与主观幸福感的相关理论和实践都有重要的意义。

关键词：社会支持、乐观、主观幸福感

\section{1. 前言}

在社会经济快速发展，高速运转的背景下，我国 的社会结构、社会体制及价值体系都处于高速发展的 状态中, 员工个体的工作强度及生活节奏均因此发生 了巨大变化, 随之而来员工的主观幸福感也产生了变 化。个体的内外部生活及工作环境发生了巨大的变化, 对员工个体的主观幸福感的影响不仅仅是员工的个 人问题, 同时也是社会问题。员工的主观幸福感成为 组织管理的重要课题, 因为它对组织的发展尤其重要, 影响个体的主观幸福感的多重因素已经引发较多学
者的关注及思考, 个体主观幸福感急需社会支持, 来 改变个体主观幸福感的状态。因此在社会快速发展的 状态下, 社会支持作为嵌入在个体工作及生活中的重 要因素之一, 是有效的增强员工的主观幸福感的重要 途径。员工个体的心理状态在快节奏高压力的工作状 态下对其主观幸福的认知有着一定的影响, 快速发展 的社会需要个体拥有乐观的心理资本来实现主观幸 福感的最大化。在经济结构快速发展情形下，社会支 持、乐观对个体有着重要的意义及作用, 从组织行为 层面上看, 个体如何获得更多的主观幸福感值得深入 探讨。 
主观幸福感 (SWB) Subjective wel1-being 是指 个体如何根据生活满意度 (认知评价) 或情感 (持续 的情感反应) 来评价自己的生活质量 (Diener et al, 2010)。个体会经历不同程度的主观幸福感, 因为 它来自个体对生活的认知和情感评价 (Diener, Lucas, \& 0shi，2002，p. 63)。根据这一理论, 即 使在恶劣的生活环境下, 人们也可以有较高的主观幸 福感。相反, 即使拥有财富、健康等优势, 一个人可 能也会拥有低水平的主观幸福感，（Seligman \& Csikszentmihalyi, 2000 \& Wei, Liao, Ku, et a1.2011）。由于主观幸福感对个人的评价及生活质量 非常重要, 学者们在向这一领域进行研究, 研究什么 样的资源可以帮助员工适应和应对工作及生活中的 挑战, 从而获得较高的主观幸福感是非常必要的。在 这样的背景下, 学者们试图寻找出哪些可用对资源可 以影响个体的主观幸福感。主要的研究从社会心理资 源的角度来解释资源是如何影响主观幸福感的 (Hobfol1, 2002)。根据这一研究趋势, 学者们提出社 会资本是个体的社会资源 (e. g. Lim \& Putnam, 2011; Siewert et al., 2011)。而心理资本作为个体的心 理资源, 会影响个体的主观幸福感 (e.g., Avey et al., 2010; Culbertson, Fullagar, \& Mills, 2010）。社会支持作为社会资本的重要因素, 社会支 持在个体的生活质量种发挥着重要的作用, 不论家庭 还是工作中, 个体在获得社会支持越多的情况下, 个 体的主观感受就越好, 自我评价的生活质量就越高, 以往的研究表明社会支持对个体的幸福感有积极的 影响（Lim \& Putnam 2010)。而个体在生活中拥有乐 观的心理资本更能够使其拥有健康乐观的精神, 更好 获取主观幸福感。

因此, 如前所述, 个体需要资源帮助他们获取良 好的生活状态, 通过本文的研究试图检验社会支持能 否满足员工个体的需求, 帮助他们来应对日常生活的 挑战以此获得更多的主观幸福感。为了发展文献, 此 外本文将社会支持与乐观联系在一起, 并考察是否通 过乐观来影响主观幸福感。

综上所述, 本研究探讨社会支持对主观幸福感的 影响, 并以乐观作为社会支持对主观幸福感的中介效 果, 期望通过弥补过去尚未被发掘的研究, 并在组织 实践应用中可以产生贡献。因此, 本研究拟进一步分 析社会支持、乐观、对主观幸福感的影响。研究框架 如下图所示。

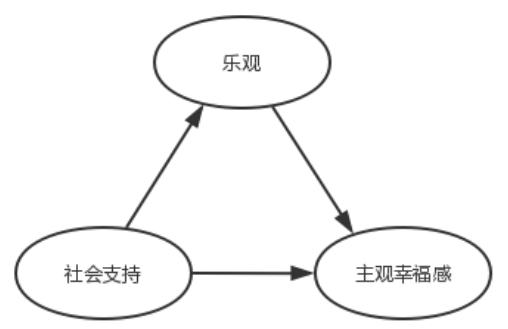

图 1 研究模型图

\section{2. 文献回顾与理论假设}

社会支持是一种重要的联结资本和人际资源, 它 将个人和他人从大致相同的社会地位, 如亲密的朋友、 家庭成员, 聚集在一起 (Irwin et al., 2008)。社会 支持在人际关系网络中受到他人的关心、尊重和重视 的一种感知, 这种感知可以通过多种途径获得 (Demaray et al., 2005)。

乐观是指做出积极的归因关于现在和未来的成 功 (Luthans, Youssef, \& Avolio, 2007, p. 3)。

主观幸福感是个体对幸福、生活满足和积极情感 的体验 (Seligman \& Csikszentmihalyi, 2000)。除 了个人的倾向性, 可用资源也会对主观幸福感产生重 大影响 (Wei et al.，2011)。社会支持和乐观是为 个体提供外部和内部资源的两种重要资源。我们将社 会支与乐观两个因素与主观幸福感联系起来 (Gorgievski et al, 2011; Hobfoll, 2002)。

\section{1. 社会支持与主观幸福感}

社会支持是个体的核心资源之一, 是一种包含情 感、信息和工具资源的应对资源 (Cohen, Gottlieb, \& Underwood, 2000; Hobfoll, 2002; Inbal et al., 2011）。个人从他或她的关系环境中获得社会支持, 例如亲密的朋友、家庭成员, 这种关系环境可以在他 们之间产生和维持社会支持 (Irwin et al.，2008)。 在这种情况下，社会支持通过关怀、相互尊重、信任 和爱有利于接受者的主观幸福感，同时缓冲接受者的 消极情绪 (Giordano \& Lindstorm, 2010; Han, 2015）。此外，社会支持提供知识和信息、服务、商 品和金钱、机会和其他建设性资源的更快传播 (Gorgievski et al., 2011; Han, 2015; Verhaeghe et al., 2012)。

支持关系中的接受者越多, 他们从社会支持给予 者那里获得的资源就越多。

因此, 我们建议

假设 1: 社会支持对主观幸福感有正向影响。

\section{2. 社会支持与乐观}

学者们认为, 心理资本是可以发展的状态, 而不 是固定的特征 (Luthans et al，2004)。乐观是心理 资本的一个重要因素, 因此, 乐观因素较少具有个性 特征, 更多的是一个可以塑造的过程。我们认为个体 的社会资源可以帮助个体获得心理资本, 即社会支持 可以帮助个体获得乐观。

乐观主义是指个体在生活的重要领域对积极和 消极结果的普遍预期, 反映了个体对积极未来的预期 能力 (Scheier \& Carver, 1985; Reker, 1997; Ho, Cheung, \& Cheung, 2010)。个人会根据自己的感知, 用乐观或悲观的解释方式来解释自己的生活 (Alan, 
2004）。这就带来了一个问题, 如何改变对个体的认 知, 保持乐观的态度。

先前的研究表明, 乐观可以受到社会背景 (即社 会支持）的影响 (Heinonen et al. , 2006; Shelby et al.，2008）。如前所述, 社会支持可以提供社会 交往伙伴感知到的可用资源, 这些资源可用于应对个 人的心理和经济压力以及抑郁症状 (Ennis et al., 2000; Shelby et a1., 2008), 有助于个体对未来有 积极的期待。因此, 我们认为, 社会支持在未来会与 乐观主义联系在一起。

假设 2：社会支持对乐观有积极影响。

\section{3. 乐观与主观幸福感}

根据 Hobfol1（2002）的主张, 个人资源包括实 物 (如家) 条件 (如婚姻状况)、精力 (如时间) 和 个人特征 (如自尊)。心理资本是个人特征的资源, 它影响着个人对自身幸福感的评价 (Avey, Luthans, Smith, \& Palmer, 2010; Siu et al., 2015; Wei et a1., 2011)。在许多实证研究中, 学者们证实了 心理资本的各个要素, 如弹乐观 (Carver et al., 2005 ) 对主观幸福感都有积极的影响。因此, 我们有 理由预测, 乐观是对主观幸福感产生积极影响的重要 资源 (Culbertson, Fullagar, \& Mi11s，2010）。

假设 3: 乐观对主观幸福感有正向影响。

\section{4. 乐观的中介作用}

在上述假设中，社会支持对乐观产生积极影响， 社会支持对主观幸福感起到正向影响的作用，乐观对 个体主观幸福感产生正向影响。基于上述假设, 有理 由认为乐观在社会支持对主观幸福感的作用产生影 响, 即中介作用。综上所述分析, 本研究提出日下假 设:

假设 4: 乐观在社会支持和主观幸福感的关系中 具有中介效应。

\section{3 研究设计}

\section{1. 样本与数据收集}

本研究以深圳地区不同行业不同的职位的员工 为调查对象, 发放问卷进行数据收集。从 2020 年 6 月 1 日开始, 至 2020 年 8 月 30 日, 共收集样本问卷 341 份, 通过对问卷进行笁选和删除, 无效问卷为 30 份, 最终确定符合研究规范的问卷为 311 份, 问卷有 效率为 $91.20 \%$ 。本研究以便利抽样的方式, 问卷发放 采用面对面直接发放和填写形式, 在进行问卷填写之 前, 均进行声明本问卷用于科学研究同时进行匿名保 密, 样本根据实际情况进行填写, 答案无对错之分, 力求真实。

\section{2. 研究工具}

本研究问卷设计采用了（Likert） 5 点量表评分 方式, 其形式为: 非常不同意为 1 , 不同意为 2 , 不 确定为 3 , 同意为 4 , 非常不同意为 5 。

社会支持参考李嵩义 (2007) 的量表进行评估, 其 中包括三个项目分别是：情感支持、工具支持、讯息 支持，每项 6 个题目，共 18 个题目。在 1 分（强烈 不同意）到 5 分 (强烈同意) 的范围内作出答复。

乐观情绪由 Scheier 和 Carver (1985) 量表评 定，该量表包括 8 项内容。样本项目是 “在不确定的 时期, 我通常期望最好的”。在 1 分（强烈不同意） 到 5 分 (强烈同意) 的范围内作出答复。在对该量表 中的消极词汇项进行评分逆转后, 对项目得分进行合 计, 得出总体乐观得分, 得分越高表示乐观程度越高。

主观幸福感采用来自 Diener 等人（2010 年）研 究的 20 个项目进行测量。员工评价自己生活的兴盛、 积极和消极。一个例子是：“我对自己的日常活动很 投入，也很感兴趣”。从 1 分 (强烈反对) 到 5 分（强 烈赞同) 的范围内都给出了热烈的回应。在 1 (从不) 到 5 (总是) 的范围内给出阳性和阴性反应。

\section{4. 研究结果分析}

\section{1. 描述性统计分析}

本研究样本有效样本共计 311 人。男性比例方 面, 男性样本总数 160 人, 占总人数 $51.44 \%$, 女性总 人数 151 人, 占总数人数比例 $48.56 \%$; 工作类型中, 高层管理者 35 人, 占总人数 $11.25 \%$, 中层管理者 76 人, 占总人数 $24.43 \%$, 基层员工 200 人, 占总人数 $64.30 \%$; 学历方面水平方面, 大专以下学历 15 人, 占总人数 $4.82 \%$, 本科学历 207 人, 占总人数 $66.56 \%$, 研究生学历 89 人, 占总人数 $28.62 \%$ 。

\section{2. 信度分析}

信度是指通过测量工具对同一事物做多次测量 时所得到的结果的一致程度。信服可以反应测量方法 和量表的可信程度。若信服系数越高, 则表明所做问 卷的稳定性及可信度越高, 本研究采用 $\alpha$ 信度系数法。 结合文献回顾及根据其它研究表明, Cronbach a 信 度係數若是大於 0.50 , 則表明問卷具有較高的可靠 性。 $\mathrm{a}$ 信度系数在 0.60 到 0.80 之间則表示可以接受 但有待优化，而小于 0.50 则被认为是不可取的。

根据本研究分析结果表明, 社会支持 a 值为 0.93 , 主观幸福感 $\alpha$ 值为 0.91 , 乐观 $\alpha$ 值为 0.92 。 通过以上数据表明本研究的问卷具有可信度, 因此本 研究的数据分析是具有相对参考价值的。 
表 1 信度分析

\begin{tabular}{lcc}
\hline 变量 & 项数 & Alpha \\
\hline 社会支持 & 18 & 0.93 \\
主观幸福感 & 20 & 0.91 \\
乐观 & 8 & 0.92 \\
\hline
\end{tabular}

\section{3. 相关分析}

本研究旨在社会支持对主观幸福感的影响, 乐观
如何社会支持和主观幸福感之间发挥中介作用。在此 研究之前, 首先要确定变量之间是否存在相关性, 本 节通过采用 Pearson 绩差相关分析方法來检验变量 之間的相关性以及相关的显著程度。如表 2 所示, 社 会支持与主观幸福感之间的相关系数为 $0.23 * *$, 表 明社会支持与主观幸福感有显著的关系。社会支持与 乐观之间的相关系数为 $0.27 * *$, 表明社会支持和乐 观之间是显著的关系。乐观和主观幸福感之间的相关 系数为 $0.45 * *$, 表明了观念和主观幸福感之间是显 著的正相关关系。

表 2 变量的均值、标准差和相关系数分析

\begin{tabular}{lccccc}
\hline & Mesn & SD & 1 & 2 & 3 \\
\hline 1. 社会支持 & 2.48 & 0.59 & - & - & $-14 * *$ \\
2. 乐观 & 2.94 & 0.79 & $0.27 * *$ & $0.23 * *$ & \\
3. 主观幸福感 & 2.21 & 0.74 & &
\end{tabular}

注:*表明 $\mathrm{P}$ 值小於 0.05 , **表明 $\mathrm{P}$ 值小於 0.01 ;

\section{4. 回归分析}

本研究利用回归分析检验乐观是否在社会支持 与主观幸福感之间发挥中介作用。由表 3 可以看出, 在模型一种 $\beta$ 值为 $0.24+(\mathrm{P}<0.1), R^{2}$ 改变量为 0.28 , 表明社会支持与主观幸福感有显著的相关性。在模型 二中, $\beta$ 值为 $0.27 *(\mathrm{P}<0.05), R^{2}$ 改变量为 0.35 , 表明社会支持与乐观有显著的相关性。在模型三中, $\beta$ 值为 $0.44(\mathrm{P}<0.01)$, 改变量为 0.10 , 表明乐观与
主观幸福感有下显著的正相关性。因此可以發現，社 会支持对主观幸福感产生正向影响。社会支持对乐观 产生正向影响。乐观对主观幸福感产生正向影响。在 模型四中, 社会支持、乐观、主观幸福感一起进行回 归分析, 发现社会支持和乐观的 $\beta$ 值分別為 $0.04(\mathrm{P}<0.1)$ 和 $0.24(\mathrm{P}<0.01), R^{2}$ 改变量为 0.38 , 对比模型一中的 $\beta$ 值 $0.24+P<(0.1)$ 有明顯的下降, 表明乐观在社会支持与主观幸福感之间发挥了中介 作用。

\section{表 3 乐观中介作用的回归分析表}

\begin{tabular}{lcccc}
\hline \multicolumn{1}{c}{ Model } & 1 & 2 & 3 & 4 \\
\hline 因数 & 主观幸福感 & 乐观 & 主观幸福感 & 主观幸福感 \\
社会支持 & $0.24+$ & $0.27^{*}$ & & 0.04 \\
乐观 & & & $0.44^{* *}$ & $0.24^{* *}$ \\
$R^{2}$ & $0.28^{* *}$ & $0.35^{* *}$ & $0.10^{* *}$ & $0.66^{* *}$ \\
$\Delta R^{2}$ & $0.28^{* *}$ & $0.35^{* *}$ & $0.10^{* *}$ & $0.38^{* *}$ \\
\hline
\end{tabular}

注: +表明 P 值小於 $0.1, *$ 表明 P 值小於 $0.05, * *$ 表明 P 值小於 0.01

\section{5. 研究结论与建议}

本研究通过文献探索, 构建了社会支持、乐观与 主观幸福感的作用机制理论模型, 并以深圳不用行业 不同职位的员工样本作为研究对象, 通过问卷采集数 据进行回归分析。结果发现: (1) 社会支持对主观幸 福感有正向影响。（2）社会支持对乐观有积极影响。 (3) 假设 3: 乐观对主观幸福感有正向影响。(4) 乐 观在社会支持和主观幸福感的关系中具有中介效应。
该研究结果对社会支持、乐观与主观幸福感的相关理 论和实践都有重要的意义。

首先, 本研究特别验证了社会支持对于员工主观 幸福感的作用起到正相关的关系。这表明, 当员工能 够获取更多的社会支持时, 对其个人的主观幸福感的 提升起到促进作用。这也说明, 组织在增强其发展的 过程中, 能够给予员工个体带来个更多的社会支持时, 对组织的发展也会起到有益的作用, 因为建议, 组织 
做好顶层设计，从更多方面可以给予员工社会支持。 其次, 本研究结果显示, 社会支持对员工主观幸福感 的影响是通过乐观这一中介来实现的。这一研究结果 说明员工个体的内在心理资本的乐观品质可以更好 的为员工带来主观幸福感, 所以建议组织注重员工个 体的心理资本建设, 通过不同的媒介增强员工个体乐 观精神，让员工提升主观幸福感。

最后, 本研究还实证了社会支持与乐观、乐观与 主观幸福感之间的关系。作为组织, 构建良好的社会 支持环境, 可以增强员工的主观幸福感。乐观的心理 状态的建立对于员工的心理健康有着重要的意义, 往 往员工个体拥有较强的乐观精神更有主观幸福感, 这 样才能够带来组织绩效的提升。

\section{REFERENCES}

[1] Carr, A. (2004). The science of happiness and human strengths. New York: Brunner-Routledge.

[2] Avey, J. B., Luthans, F., Smith, R. M., \& Palmer, N. F. (2010). Impact of positive psychological capital on employee well-being over time. Journal of occupational health psychology, 15(1), 17.

[3] Carver, C. S., Smith, R. G., Antoni, M. H., Petronis, V. M., Weiss, S., \& Derhagopian, R. P. (2005). Optimistic personality and psychosocial well-being during treatment predict psychosocial well-being among long-term survivors of breast cancer. Health Psychology, 24(5), 508.

[4] Lim, C., \& Putnam, R. D. (2010). Religion, social networks, and life satisfaction. American sociological review, 75(6), 914-933.

[5] Cohen, S., \& Hoberman, H. M. (1983). Positive events and social supports as buffers of life change stress 1 . Journal of applied social psychology, 13(2), 99-125.

[6] Culbertson, S. S., Fullagar, C. J., \& Mills, M. J. (2010). Feeling good and doing great: The relationship between psychological capital and well-being. Journal of occupational health psychology, 15(4), 421.

[7] Culbertson, S. S., Fullagar, C. J., \& Mills, M. J. (2010). Feeling good and doing great: The relationship between psychological capital and well-being. Journal of occupational health psychology, 15(4), 421.

[8] Demaray, M. K., Malecki, C. K., Davidson, L. M., Hodgson, K. K., \& Rebus, P. J. (2005). The relationship between social support and student adjustment: A longitudinal analysis. Psychology in the Schools, 42(7), 691-706.
[9] Diener, E., Wirtz, D., Tov, W., Kim-Prieto, C., Choi, D. W., Oishi, S., \& Biswas-Diener, R. (2010). New well-being measures: Short scales to assess flourishing and positive and negative feelings. Social indicators research, 97(2), 143156.

[10] Diener, E., Lucas, R. E., \& Oishi, S. (2002). Subjective well-being: The science of happiness and life satisfaction. Handbook of positive psychology, 2, 63-73.

[11] Ennis, N. E., Hobfoll, S. E., \& Schröder, K. E. (2000). Money doesn't talk, it swears: How economic stress and resistance resources impact inner-city women's depressive mood. American Journal of Community Psychology, 28(2), 149173.

[12] Heinonen, K., Räikkönen, K., Matthews, K. A., Scheier, M. F., Raitakari, O. T., Pulkki, L., \& Keltikangas-Järvinen, L. (2006). Socioeconomic status in childhood and adulthood: Associations with dispositional optimism and pessimism over a 21-year follow-up. Journal of personality, 74(4), 1111-1126.

[13] Hobfoll, S. E. (2002). Social and psychological resources and adaptation. Review of general psychology, 6(4), 307-324.

[14] Hobfoll, S. E. (2002). Social and psychological resources and adaptation. Review of general psychology, 6(4), 307-324.

[15] Irwin, J., LaGory, M., Ritchey, F., \& Fitzpatrick, K. (2008). Social assets and mental distress among the homeless: Exploring the roles of social support and other forms of social capital on depression. Social Science \& Medicine, 67(12), 1935-1943.

[16] Lim, C., \& Putnam, R. D. (2010). Religion, social networks, and life satisfaction. American sociological review, 75(6), 914-933.

[17] Luthans, F., Luthans, K. W., \& Luthans, B. C. (2004). Positive psychological capital: Beyond human and social capital.

[18] Scheier, M. F., \& Carver, C. S. (1985). Optimism, coping, and health: assessment and implications of generalized outcome expectancies. Health psychology, 4(3), 219.

[19] Seligman, M. E., Steen, T. A., Park, N., \& Peterson, C. (2005). Positive psychology progress: empirical validation of interventions. American psychologist, 60(5), 410.

[20] Shelby, R. A., Crespin, T. R., Wells-Di Gregorio, 
S. M., Lamdan, R. M., Siegel, J. E., \& Taylor, K. L. (2008). Optimism, social support, and adjustment in African American women with breast cancer. Journal of Behavioral Medicine, 31(5), 433-444.

[21] Siewert, K., Antoniw, K., Kubiak, T., \& Weber, H. (2011). The more the better? The relationship between mismatches in social support and subjective well-being in daily life. Journal of Health Psychology, 16(4), 621-631.

[22] Wei, M., Liao, K. Y. H., Ku, T. Y., \& Shaffer, P. A. (2011). Attachment, self-compassion, empathy, and subjective well-being among college students and community adults. Journal of personality, 79(1), 191-221. 